\title{
Vortex-MEMS filters for wavelength-selective orbital-angular-momentum beam generation
}

Paul, Sujoy; Lyubopytov, Vladimir; Schumann, Martin F.; Cesar, Julijan; Malekizandi, Mohammadreza; Haidar, Mohammad T.; Porfirev, Alexei P.; Gurbatov, Stanislav O.; Wegener, Martin; Chipouline, Arkadi Total number of authors:

14

Published in:

Proceedings of SPIE

Link to article, DOI:

10.1117/12.2252494

Publication date:

2017

Document Version

Publisher's PDF, also known as Version of record

Link back to DTU Orbit

Citation (APA):

Paul, S., Lyubopytov, V., Schumann, M. F., Cesar, J., Malekizandi, M., Haidar, M. T., Porfirev, A. P., Gurbatov, S. O., Wegener, M., Chipouline, A., KÃ¹/4ppers, F., Andrews, D. L. (Ed.), Galvez, E. J. (Ed.), \& Glückstad, J. (Ed.) (2017). Vortex-MEMS filters for wavelength-selective orbital-angular-momentum beam generation. In Proceedings of SPIE (Vol. 10120). [101200G] SPIE - International Society for Optical Engineering. Proceedings of SPIE - The International Society for Optical Engineering https://doi.org/10.1117/12.2252494

\section{General rights}

Copyright and moral rights for the publications made accessible in the public portal are retained by the authors and/or other copyright owners and it is a condition of accessing publications that users recognise and abide by the legal requirements associated with these rights.

- Users may download and print one copy of any publication from the public portal for the purpose of private study or research.

- You may not further distribute the material or use it for any profit-making activity or commercial gain

- You may freely distribute the URL identifying the publication in the public portal 


\title{
Vortex-MEMS filters for wavelength-selective orbital-angular-momentum beam generation
}

\author{
Sujoy Paul ${ }^{a}$, Vladimir S. Lyubopytov ${ }^{a, b}$, Martin F. Schumann ${ }^{c, d}$, Julijan Cesar ${ }^{a}$, \\ Mohammadreza Malekizandi ${ }^{a}$, Mohammad T. Haidar $^{a}$, Alexei P. Porfirev ${ }^{e, f}$, Stanislav O. \\ Gurbatov $^{g}$, Martin Wegener ${ }^{c, d}$, Arkadi Chipouline ${ }^{a}$, and Franko Küppers ${ }^{a}$ \\ ${ }^{a}$ Technische Universität Darmstadt, Institute for Microwave Engineering and Photonics \\ Merkstrasse 25, Darmstadt, Germany; \\ ${ }^{b}$ Technical University of Denmark (DTU), Department of Photonics Engineering \\ Ørsteds Plads 343, 2800 Kgs. Lyngby, Denmark; \\ ${ }^{c}$ Karlsruhe Institute of Technology (KIT), Institute of Nanotechnology \\ 76021 Karlsruhe, Germany; \\ ${ }^{d}$ Karlsruhe Institute of Technology (KIT), Institute of Applied Physics \\ 76128 Karlsruhe, Germany; \\ e Samara National Research University, Moskovskoye shosse 34, Samara 443086, Russia; \\ ${ }^{f}$ Image Processing Systems Institute of the Russian Academy of Sciences \\ Molodogvardeiskaya St. 151, Samara 443001, Russia; \\ ${ }^{g}$ Far Eastern Federal University, 8 Sukhanova Str., Vladivostok 690041, Russia
}

\begin{abstract}
In this paper an on-chip device capable of wavelength-selective generation of vortex beams is demonstrated. The device is realized by integrating a spiral phase-plate onto a MEMS tunable Fabry-Perot filter. This vortex-MEMS filter, being capable of functioning simultaneously in wavelength and orbital angular momentum (OAM) domains at around $1550 \mathrm{~nm}$, is considered as a compact, robust and cost-effective solution for simultaneous OAM- and WDM optical communications. Experimental spectra for azimuthal orders 1,2 and 3 show OAM state purity $>92 \%$ across $30 \mathrm{~nm}$ wavelength range. A demonstration of multi-channel transmission is carried out as a proof of concept.
\end{abstract}

Keywords: Micro-elctro-mechanical system (MEMS), surface micromachining, electrothermal tuning, vortex, optical angular momentum (OAM), optical data transmission

\section{INTRODUCTION}

Escalating data-transmission capacity has been an intensive research topic for a while. This includes investigating different physical properties of lightwave such as amplitude, phase, wavelength and polarization. The well-known techniques for increasing data channels include wavelength-/polarization- and space-division Multiplexing (WDM/PDM or SDM), respectively. A special case of SDM comes into application by employing the orthogonal spatially overlapping and co-propagating spatial modes, known as mode-division multiplexing (MDM). There are several different types of orthogonal modal basis sets of that are potential candidates for such MDM systems. Orbital angular momentum (OAM) is specially interesting among them, primarily due to its theoretically unlimited orthogonal basis of information carriers which is supported by the fact that a good number of its states can be readily realized in the laboratory. ${ }^{1}$

It is only in 1992 that Allen recognized that any helically phased light beam with an azimuthal term $\exp (\mathrm{i} l \varphi)$, commonly known as optical vortex, inherently possesses an OAM of $l \hbar$ per photon (where integer $l$ is the

Further author information: Send correspondence to Sujoy Paul E-mail: paul@imp.tu-darmstadt.de, Telephone: +49 6151 16-28430 


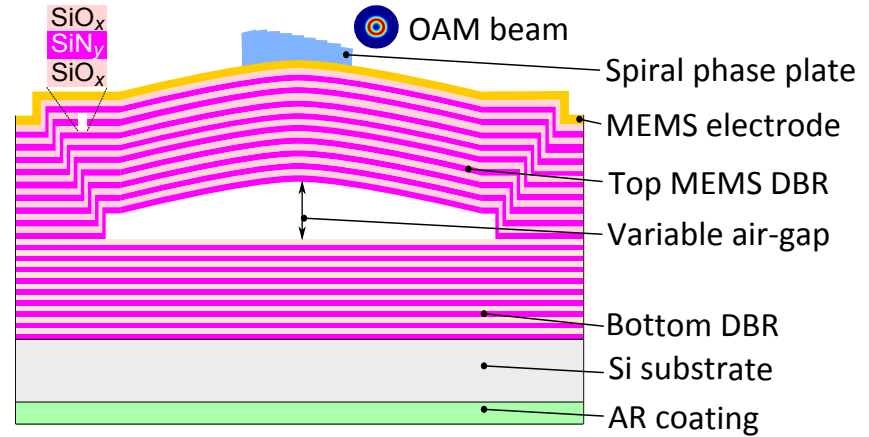

(a)

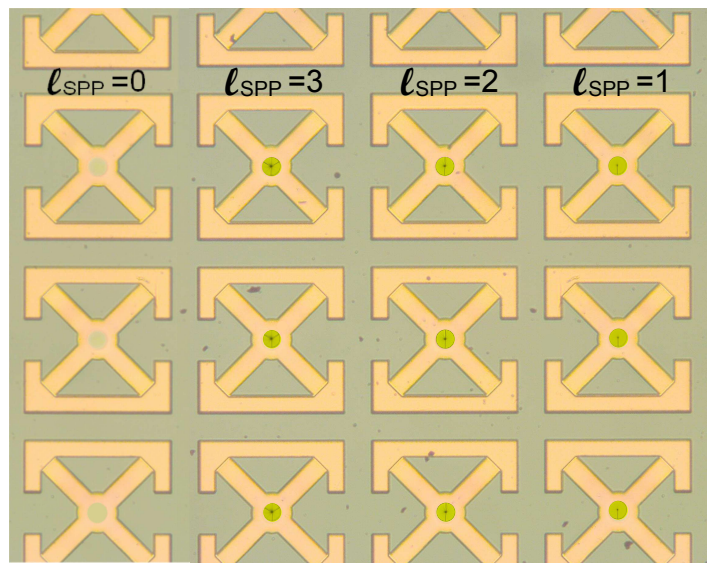

(b)

Figure 1: (a) Cross-section of a vortex-MEMS Fabry-Perot filter. (b) Optical images of SPPs of orders 1, 2 and 3 , fabricated directly on MEMS in 2-D array.

topological charge, $\varphi$ is the azimuthal angle, and $\hbar$ is the Planck constant). ${ }^{2}$ In contrast to spin angular momentum which has only two possible polarization states $\pm \hbar$, OAM can provide an infinite range of states due to its potentially unlimited values of $l$. In current realizations of OAM-based transmission systems the generation, multiplexing and detection of optical vortex is predominantly provided by discrete, bulk optical devices, such as liquid crystal based spatial light modulators ${ }^{3}$ or separate phase plates. ${ }^{4}$ Such approaches require operations with free space beams as well as precise alignment of the elements, thus cannot provide robustness and repeatability. Hence, development of the compact, on-chip integrated optical components for generation, processing and detection of vortices is the next significant step towards robust, energy- and cost-effective OAMbased communication systems. Recently vertical-cavity surface-emitting laser (VCSEL) based direct vortex generation has been demonstrated, ${ }^{5}$ where a dielectric stepped spiral phase plate (SPP) has been deposited on the aperture of a single-mode (SM) $860 \mathrm{~nm}$ device. However, for future communications based on the converged OAM- and WDM devices capable of functioning simultaneously in both wavelength and OAM domains would be of key importance. Moreover, for communication applications vortex generation at the long-wavelength regime $(1300-1600 \mathrm{~nm})$ would be preferred.

In this paper we propose to multiply the capacity of current optical communications by combining OAMmultiplexing with WDM technique. In order to realize this idea, an innovative approach of generating wavelengthtunable OAM-carrying beams by integrating micro-sized SPPs on microelectromechanical system (MEMS) tunable Fabry-Perot optical filter has been reported. By integrating filters with mass-manufacturable SPPs, highpurity OAM modes and their superposition states could be generated while maintaining advantages in cost and power efficiency. This device is capable of functioning simultaneously in both wavelength and OAM domains at telecom wavelengths: it simultaneously separates wavelengths in a tunable fashion, while splitting vortices of different fixed orders. Fabrication of MEMS filters utilizes surface micromachining technology, suitable for mass fabrication in 2-D arrays. Thus, by integrating filters with mass-manufacturable SPPs, high-purity OAM modes and their superposition states could be generated while maintaining advantages in cost and power efficiency. In this paper we show that our proposed vortex-MEMS filter construction can be used as a simultaneous WDM and OAM demultiplexer. We consider this compact, on-chip device to be especially useful for communication applications, where the distances are short but high data rates are desirable, such as data center networks ${ }^{6}$ and optical interconnects. ${ }^{7}$

\section{DEVICE STRUCTURE AND FABRICATION}

A wavelength-tunable vortex-MEMS filter is basically a Fabry-Perot MEMS resonator with an integrated SPP, as shown in Fig. 1. A fully fabricated device comprises an anti-reflection coating (ARC), a Si substrate, a fixed bottom distributed Bragg reflector (DBR), a variable air-gap, a movable MEMS DBR and a SPP. First, an 

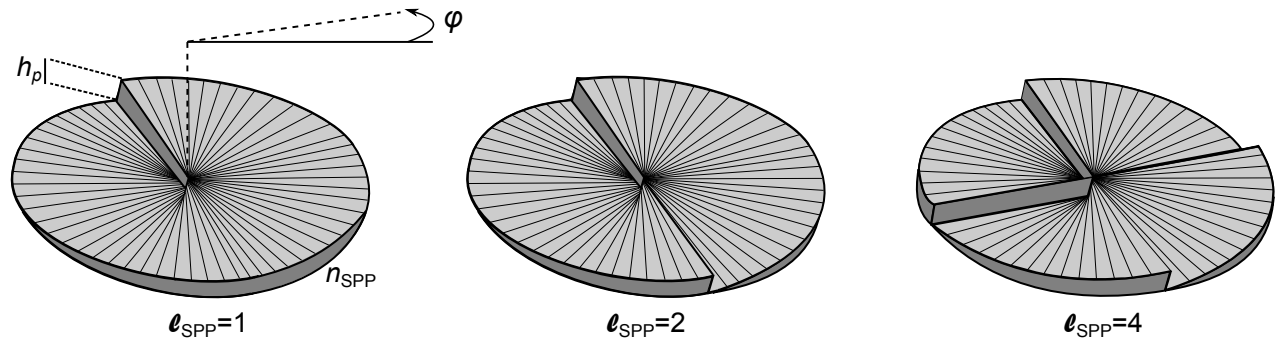

Figure 2: Three different orders of SPPs.

SiON ARC of $\lambda_{0} / 4$ ( $\lambda_{0}$ being the target centre wavelength) thickness is deposited in a low-temperature PECVD chamber. The bottom DBR comprising nine pairs of $\mathrm{SiO}_{x} / \mathrm{SiN}_{y}$ dielectric layers in an alternating sequence are deposited on the other side of the wafer. To incorporate appox. $5 \mu \mathrm{m}$ of air-gap, a Ni sacrificial layer is sputtered and laterally structured with lithography and wet-chemical etching. Then the layers of the MEMS DBR are deposited with the same material sequence. However, an intrinsic stress gradient is incorporated intentionally in the MEMS dielectric materials for the membrane to bend concavely after the sacrificial layer is completely removed. Each DBR layer has an optical thickness of $\lambda_{0} 4$, resulting in a total thickness of $5.5 \mu \mathrm{m}$. With a refractive index difference between $\mathrm{SiO}_{x}$ and $\mathrm{SiN}_{y}$ of $\Delta n=0.5$, the MEMS mirror exhibits a reflectivity $>99.5 \%$ over a broadband wavelength range of $120 \mathrm{~nm}$ around the center wavelength of $1550 \mathrm{~nm}$. On top of the MEMS DBR a $\mathrm{Cr} / \mathrm{Au}$ actuation electrode is evaporated. To accommodate the SPP, a circular opening in the $\mathrm{Cr} / \mathrm{Au}$ layer is structured. Afterwards, the top DBR is covered with a $\mathrm{Ni}$ etch mask and dry etched to achieve the characteristic shape. Finally, both sacrificial layer and the etch mask are wet etched and the device is dried using a critical point drier. For an elaborated description of the structure and fabrication of the MEMS mirror, the readers are referred to. ${ }^{8,9}$ The resonant wavelength can be tuned either by electro-thermal actuation of the MEMS or just by changing the substrate temperature. As the suspension beams of the MEMS are fixed to fixed DBR surface, it can expand only in upward direction. Thus the optical cavity length is increased, resulting in a red-shift in the resonance wavelength. In contrast, a blue-shift of emission is prominent in thermal tuning scheme due to a slower thermal expansion of the MEMS compared to the Si substrate.

In the following step, the nanoscribe Photonic Professional 3-D lithography system is employed to write the SPPs on the MEMS. ${ }^{10}$ The SPP is fabricated utilizing direct laser writing of polymers (Photonic Professional GT with IP-Dip photoresist, nanoscribe GmbH, Germany) in dip-in configuration to enable integration directly on the surface of the finished MEMS device. First, the chip is fully covered by the liquid dip-in photoresist. This allows for convenient writing on top of opaque substrates. The photoresist is designed such that single

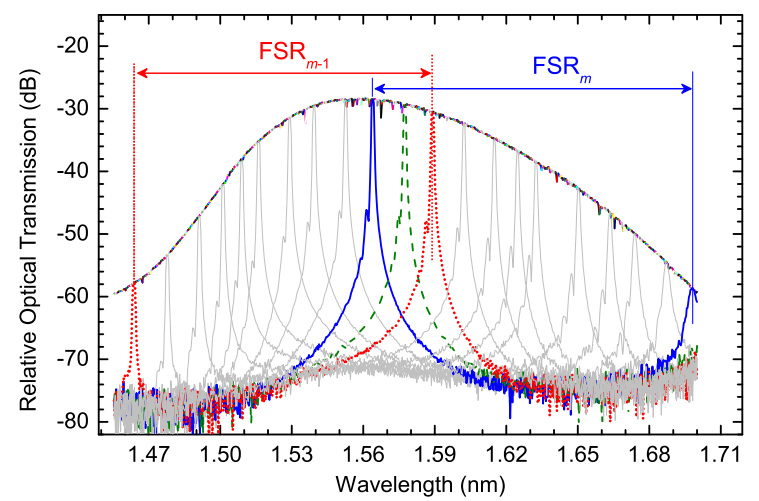

(a)

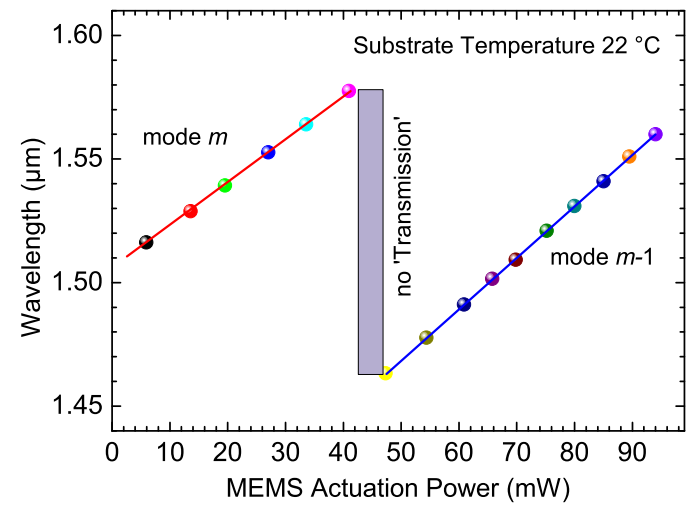

(b)

Figure 3: (a) Dependence of MEMS-filter transmittance of the resonance wavelength. (b) wavelength as a funtion of MEMS actuation power. 
photons from the writing laser cannot be absorbed, but a two-photon absorption can induce polymerization. Since nonlinear processes scale with intensity, the polymerization occurs where the intensity is the highest, i.e., in the focal volume of the laser. Furthermore, the polymerization process exhibits a threshold behavior. Thus, only a volume within a certain iso-intensity surface given by the threshold intensity of the resist is polymerized. Thus, an $l$-fold SPP is then printed on the aperture of the MEMS DBR. The estimated height $h_{p}$ of the SPP depends on the azimuthal angle $\varphi$ according to

$$
h_{p}(\varphi)=\frac{\varphi l_{p} \lambda_{0}}{2 \pi\left(n_{p}-n_{0}\right)},
$$

where $l_{p}$ is the required topological charge of the phase plate, $n_{p}$ and $n_{0}$ are the refractive indices of the material of the SPP and the surrounding medium (air), respectively. While the design height depends continuously on $\varphi$, the mask is divided into 60 discrete steps. Consequently, the geometry can be sliced into layers of equal height which can be exposed one after another using the rapid galvo-scanning technique. It is worth mentioning that the fabrication of a $52 \mu \mathrm{m}$-diameter SPP takes only approx. 5 minutes. An illustration of three different orders of SPPs is shown in Fig. 2.

Figure 3(a) shows filter transmittance of the resonance wave-length when the filter is tuned using the electrothermal actuation scheme. As can be seen, with an increasing MEMS current, the spectra is red-shifted. The shape of the envelope follows the trace of the semiconductor optical amplifier (SOA). The MEMS-filter shows a full-width at half-maximum (FWHM) bandwidth of $0.2 \mathrm{~nm}$ and a free spectral range (FSR) of $126 \mathrm{~nm}$. The FSR is defined by the spectral spacing between two consecutive modes is tone of the limiting factors (the other is the reflectivity bandwidth of the DBRs) for a mode-hop free tuning. It can altered by designing an optimum air-gap by changing the stress gradient of the top DBR and reflectivity of the DBR layers. The bandwidth of the MEMS-filter does not change after integrating the SPP mask on its upper DBR. Figure 3 (b) shows the emission wavelength as a funtion of MEMS actuation power, where two consecutive modes can be seen (in this case no mode-hop will occur, if the MEMS current is set in the beginning of mode $m-1$ ). MEMS filters with SPPs show only slight extra losses at the resonance wavelength compared to the MEMS filter without SPP. This difference, reliably measured for the filter with $l_{\mathrm{SPP}}=1$, amounts to $1.93 \mathrm{~dB}$ and can be readily explained by the additional absorption and scattering of the light by the SPP itself.

\section{EXPERIMENT SETUP FOR OAM BEAM GENERATION AND DETECTION}

In order to reduce the complexity of experimental setup the simple thermal heating approach is applied for filter tuning, which allows smaller tuning range compared with the case of electro-thermal MEMS actuation.

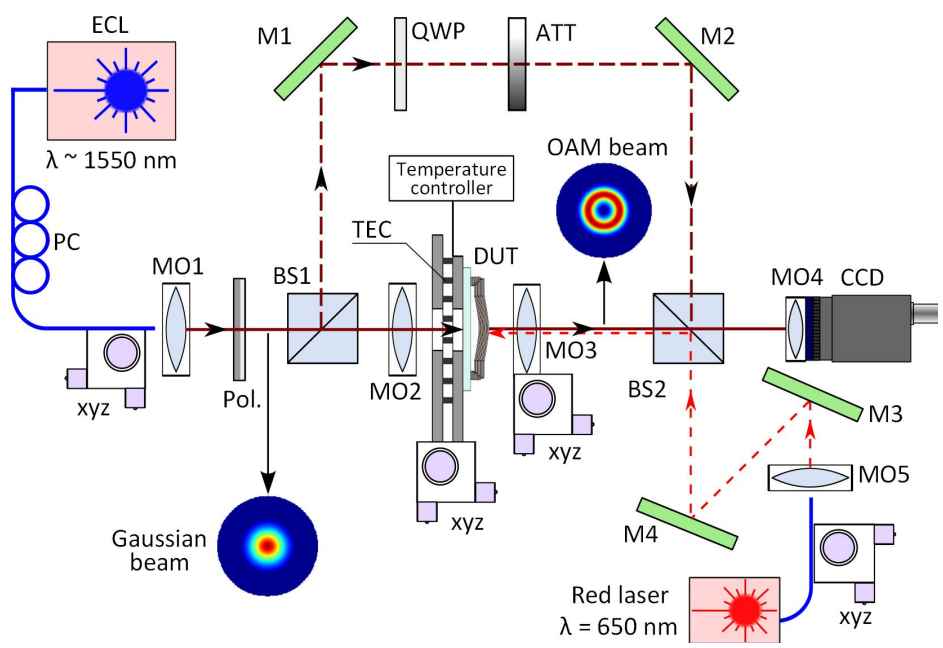

Figure 4: The experimental setup for generation, detection and characterization of the OAM modes. 


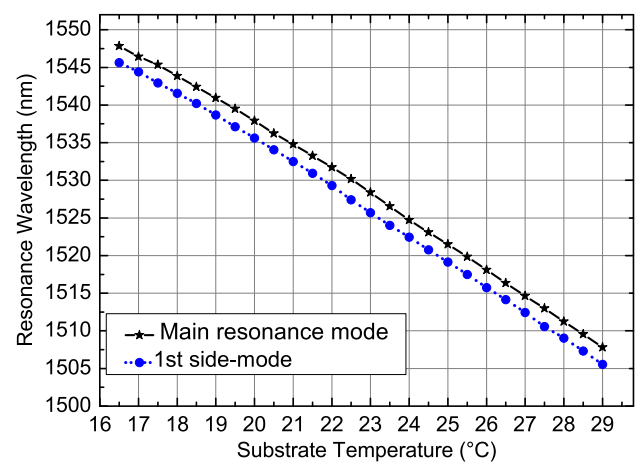

Figure 5: Tuning wavelength as a function of wafer temperature.

However, even with this simple approach the tuning range of more than $30 \mathrm{~nm}$ can be achieved. Figure 4 shows the experimental setup for testing the functionality of the proposed vortex-MEMS filters. The device under test (DUT) is fed by a tunable external cavity laser (ECL), which allows scanning the supported wavelength range for the maximums of transmittance. Microscope objective MO1 collimates the fiber output from ECL. Polarizer provides linear polarization of the beam at the input of the DUT, and the fiber polarization controller (PC) installed at the output of ECL allows optimizing the state of polarization to maximize the power after the polarizer. Phase profile analysis of the beams transmitted through the vortex-MEMS filter is implemented using a Mach-Zehnder interferometer, which allows capturing the fringe patterns between the beam emitted from the DUT and the reference flat-wavefront Gaussian beam. To reconstruct the phase distributions from fringe patterns, an approach with parallel interference between the OAM beam and a reference beam is applied. ${ }^{11}$ This approach implies capturing two fringe patterns for each phase profile measurement, one of which is obtained with the additional $\pi / 2$ phase shift between the paths of interferometer. ${ }^{11,12}$ Beam splitter BS1 and mirrors M1, M2 prepare the reference beam parallel to the OAM beam, both are then combined with BS2 to obtain fringe patterns on the IR CCD-camera. The variable attenuator ATT is used to make the power of the reference beam comparable with that of the beam, emitted from the filter. As the beam propagating through the Mach-Zehnder interferometer is already linearly polarized, the quarter wave plate allows introducing the additional $\pi / 2$ phase shift between interfering beams by aligning its fast or slow axis with the electric field vector of the incident light. Microscope objective MO3 provides matching the beam waist with the output aperture and the curvature of the MEMS mirror. In order to apply the phase front shaped by SPP to the beam emitted from the filter, its input beam is incident upon the wafer from the side of the substrate. For preliminary alignment of the DUT with the input IR beam, the fiber coupled red light laser is used, which points the aperture of a definite DUT on the upper side of the wafer. The beam from the red light laser, previously aligned with the IR beam by means of mirrors M3, M4, is also transmitted through the beam splitter BS2. Final precise positioning of the DUT and optimization of the incidence angle of the input beam is provided by controlling the output power and the "doughnut" intensity profile of the beam emanated from the filter. To capture the intensity profiles and fringe patterns a phosphor-coated NIR CCD-camera is used. Microscope objectives MO4 and MO5 provide magnification of the image to be captured by the camera. Tuning of the filters is realized by controlling the substrate temperature with a thermoelectric cooler (TEC) element.

Figure 5 shows the typical dependence of the filter resonance wavelength on the wafer temperature, which is seen to be almost linear. The main mode (stars) of the Fabry-Perot resonator is Gaussian in the case of simple MEMS filter (without SPP) and possesses the highest intensity, whereas the 1st side-mode (circles) is defined as the mode with second highest intensity after the main mode. The transmittance of MEMS filters with the SPPs of different azimuthal orders as well as of the simple MEMS filter without SPP have been measured over a continuous tuning range, limited by the simple thermal heating approach applied in this setup in order to reduce its complexity.

The dependence of the transmittance on the resonant wavelength for the main mode of MEMS filters are shown in Fig. 6. The output power of the modes emitted through the filters is estimated in free space from the camera images in order to avoid the influence of non-optimal coupling of higher-order modes with the fiber on the 

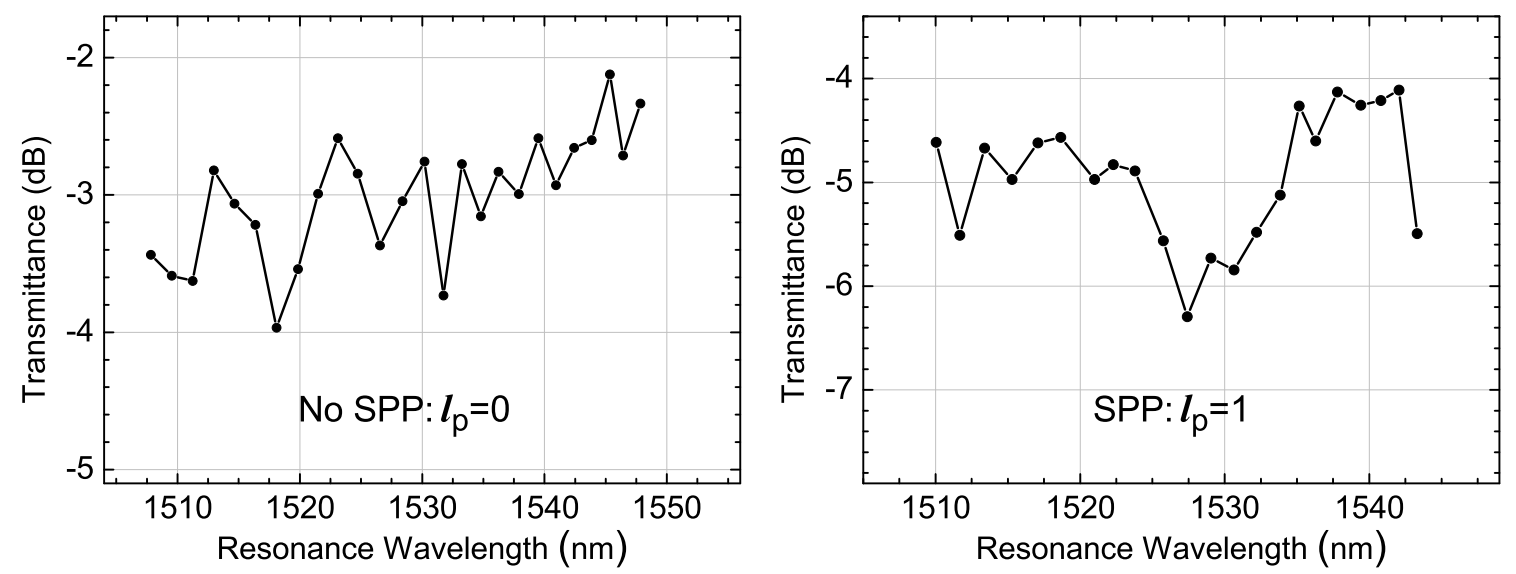

(a)

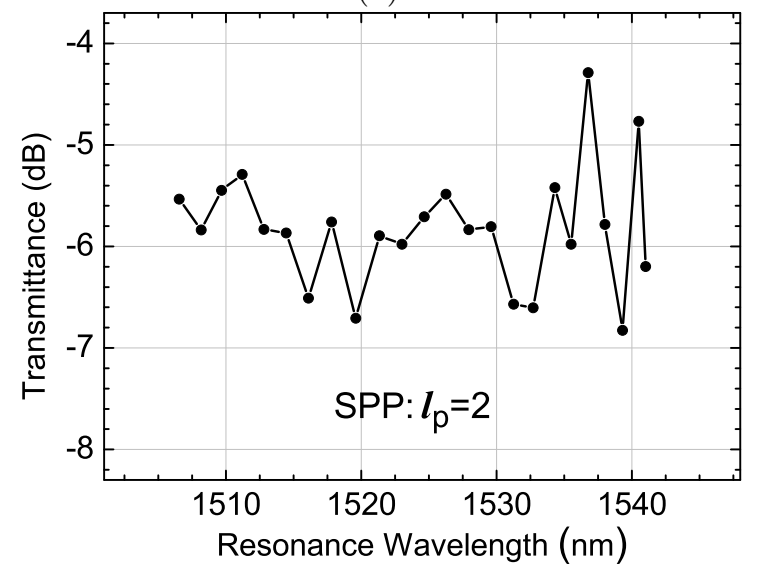

(c)

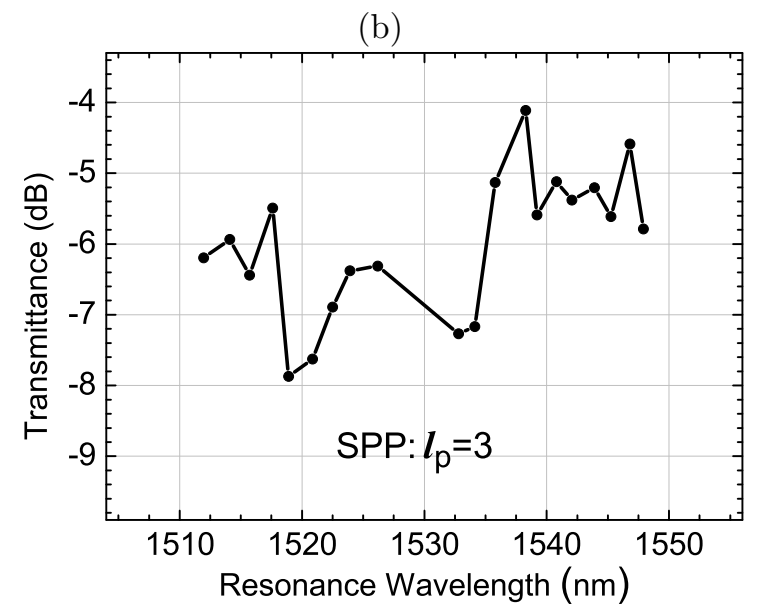

(d)

Figure 6: Transmittance of the SPP modes with $l=0,1,2$ and 3 as a function of resonance wavelength.

power measurement results. To obtain correct power estimations, the nonlinearity of the camera response and the wavelength dependence of the camera output have been taken into account. For each device the transmittance fluctuates to a small extent around some average value (cf. Table 1) and does not demonstrate any significant decay over the whole measured range of wavelengths. However, it can be seen that the average transmittance of the vortex-MEMS filters is to some extent lower than that of the MEMS-filter without SPP. For the filter with SPP $l_{p}=1$ this difference is $1.93 \mathrm{~dB}$. This fact can be readily explained by the additional absorption and scattering of the light by the SPP itself. As the SPPs of different azimuthal orders have the same construction and their average thickness is also the same, the lower values of transmittance obtained for the devices with $l_{p}=2$ and $l_{p}=3$ can be explained by the methodological inaccuracy when measuring the power with the camera,

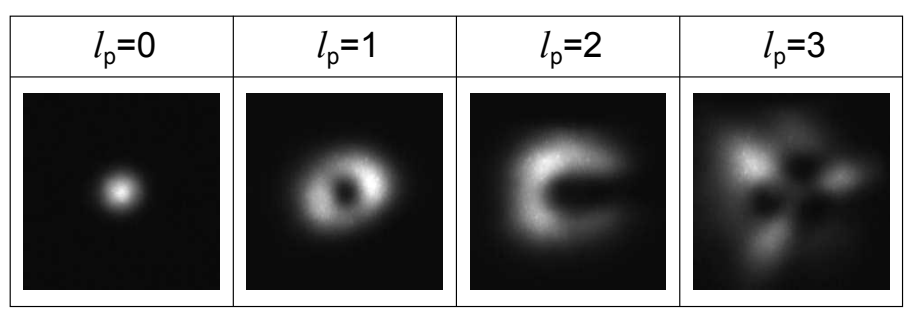

Figure 7: Intensity distributions for the main resonance mode of the vortex-MEMS filters with the 4 azimuthal orders. 


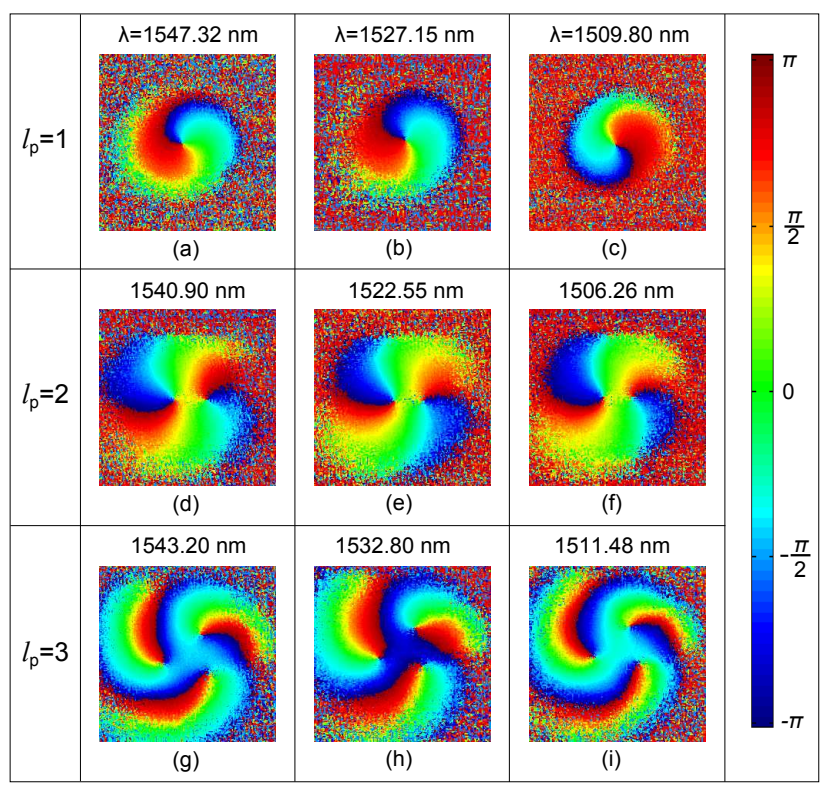

Figure 8: Measured phase distributions for the main resonance mode of the vortex-MEMS filters with the 3 azimuthal orders $\left(l_{p}=1,2,3\right)$ at different resonance wavelengths.

as the higher order of OAM mode leads to the broader transversal field distribution, and as a result some part of beam energy cannot be distinguished from the noise level due to the limited sensitivity of the camera.

Also the transmittance for the side-modes have been estimated, which seems to be dependent dramatically on the input beam alignment. When capturing images of the beams transmitted through the MEMS-filters, input beam alignment is kept in order to maximize the transmittance for the main resonator mode. The transmittances for the 1st side-modes averaged over the measured tuning ranges are also shown in Table 1. However, for the most practical cases these values of side-mode transmittance captured for the free space radiation are not important. For example, if the vortex-MEMS filter is used as an OAM- and wavelength-selective device for detecting channel signals in a OAM-wavelength-multiplexed transmission system, only the received mode corresponding to the opposite azimuthal order of SPP at the main resonant wavelength of MEMS-filter can be transformed to the Gaussian mode and coupled to the SM fiber. In the case of integration of such MEMS-resonator with the VCSEL for wavelength-tunable direct vortex generation, the resulting side-mode suppression ratio (SMSR) will be defined mostly by the optimal coupling of the main resonator mode with the active media.

Figure 7 shows the intensity distributions of the main resonator mode captured at the output of simple MEMS-filter and those with SPPs of azimuthal orders $l_{p}=1,2,3$. The shown images correspond to the resonant wavelengths near $1540 \mathrm{~nm}$. As there is no visible difference in the shape of intensity distributions obtained for each device over the tested wavelength range (except the overall beam intensity), for the sake of brevity only intensity distributions for one wavelength are shown. Phase distributions extracted from the captured fringe patterns are shown in Fig. 8, where for each device three phase distributions corresponding to different wavelengths (lower, upper and center of the tuning range) are represented. However, the beams at the output of vortex-MEMS filters of azimuthal orders $l_{p}=2$ and $l_{p}=3$ have non-uniform intensity distribution over the "doughnut" mode profile and the corresponding non-symmetry in the phase distributions. Noteworthy, this peculiarity does not change

Table 1: Averaged transmittance of the vortex-MEMS filters.

\begin{tabular}{c|c|c|c|c} 
& \multicolumn{4}{|c}{ Transmittance (dB) } \\
\hline Vortex type & no SPP & $l_{p}=1$ & $l_{p}=2$ & $l_{p}=3$ \\
\hline Main mode & -3.01 & -4.94 & -5.83 & -6.01 \\
\hline 1st side-mode & -15.14 & -14.59 & -12.78 & -13.88
\end{tabular}



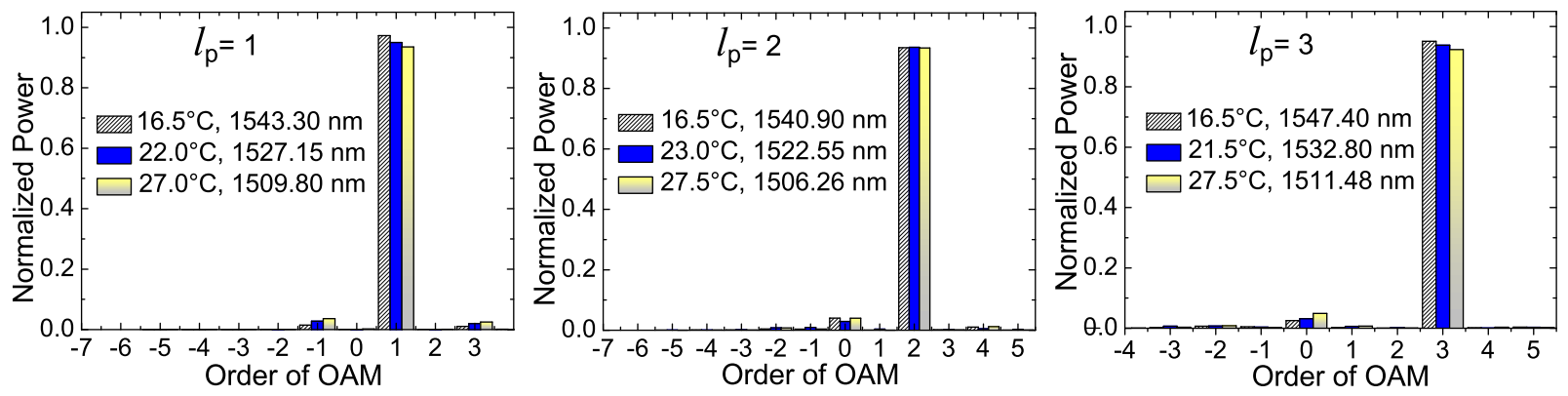

Figure 9: Normalized power of OAM states in the generated vortex beams.

with the temperature and the corresponding resonant wavelength. Therefore, these non-ideal complex amplitude distributions can be explained by non-ideal symmetry of upper MEMS DBR convexity resulting in a deviation of the center of the transmitted beam from the geometrical center of MEMS filter aperture. As the SPP is aligned to the geometrical center of the filter aperture, such behavior of the filter leads to some displacement between the output beam and SPP.

Figure 9 shows the normalized OAM spectra, obtained by expanding the experimental phase distributions (see Fig. 8) in terms of angular harmonics $\exp (i l \varphi)$. Two possible factors affecting the purity of the generated OAM states are: i) phase profile distortion due to output beam displacement, which is present for the whole range of resonant wavelengths, and ii) reduction of the efficiency of mode conversion from the Gaussian mode to the OAM modes as the resonance wavelength goes down from the designed SPP wavelength of $1550 \mathrm{~nm}$. But the tunability band is relatively small compared to the central wavelength, and as a result the OAM state purity degrades only by 0.0373 (from 0.9726 to 0.9353 ) over the wavelength range of $33.60 \mathrm{~nm}$ for the device with SPP $l_{p}=1$, and by 0.0279 (from 0.9512 to 0.9233 ) over the wavelength range of $35.92 \mathrm{~nm}$ for the device with $\mathrm{SPP} l_{p}=3$. As to the device with SPP $l_{p}=2$, there is no significant improvement of OAM beam quality when increasing the wavelength (the normalized power of OAM $l_{p}=2$ proves to be 0.9353 at $1540.9 \mathrm{~nm}$ and 0.9336 at $1506.26 \mathrm{~nm}$ ), which can be explained by the technological properties of current device or by an inaccuracy when measuring the phase distributions. On the whole, as can be seen from Fig. 8, the output beams retain their vortical structure over the whole considered wavelength range, and even for the vortex-MEMS filter of azimuthal order $l_{p}=3$ the normalized power of OAM state $l_{p}=3$ is not lower than 0.9233 .

\section{SETUP FOR DATA TRANSMISSION EXPERIMENTS}

In order to perform wavelength- and OAM-multiplexed transmission tests, a prototype of an optical communication system was assembled, consisting of a typical fiber optic transmitter with a WDM multiplexer (WDM-MUX), free-space OAM multiplexer (OAM-MUX), and receiver, where simultaneous demultiplexing of wavelength and spatial channels is provided by the MEMS-filter see Fig. 10. Two tunable ECLs feed WDM-MUX generating two information channels with a variable wavelength difference. One of the wavelength channels is considered as primary, i.e. carrying the useful signal to be received, while another one is considered as secondary, i.e. carrying another signal, which performs as a disturbance. BER measurements have been performed with the primary channel only. After passing polarization controllers PC1 and PC2 the laser signals is independently modulated by Mach-Zehnder modulators (MZMs) for on-off keyying (OOK). The modulator is driven by pseudorandom bit sequences (PRBS) of lengths of $2^{31}-1$ and $2^{23}-1$ bits at $10 \mathrm{Gbit} / \mathrm{s}$ electrical signals, respectively. Modulators MZM1 and MZM2 provide extinction ratios of 9.2 and $9.46 \mathrm{~dB}$, respectively. Being combined by a 50/50 coupler and pre-amplified using an erbium-doped fiber amplifier (EDFA), $95 \%$ of the WDM signal are coupled to the input of OAM-MUX. Another 5\%-part of the generated WDM signal is split between the p-i-n photodiode (PIN ) (95\%) and optical spectrum analyzer (OSA) (5\%). By connecting the digitizing oscilloscope to the output of PIN, initial eye-diagrams of individual WDM-channels can be captured in order to optimize performance. OSA serves to monitor the spectrum of the generated WDM signals in order to ensure that WDM channels have equal powers and are separated with a desirable $\Delta \lambda$. The total output power of EDFA is kept at the level of 20 


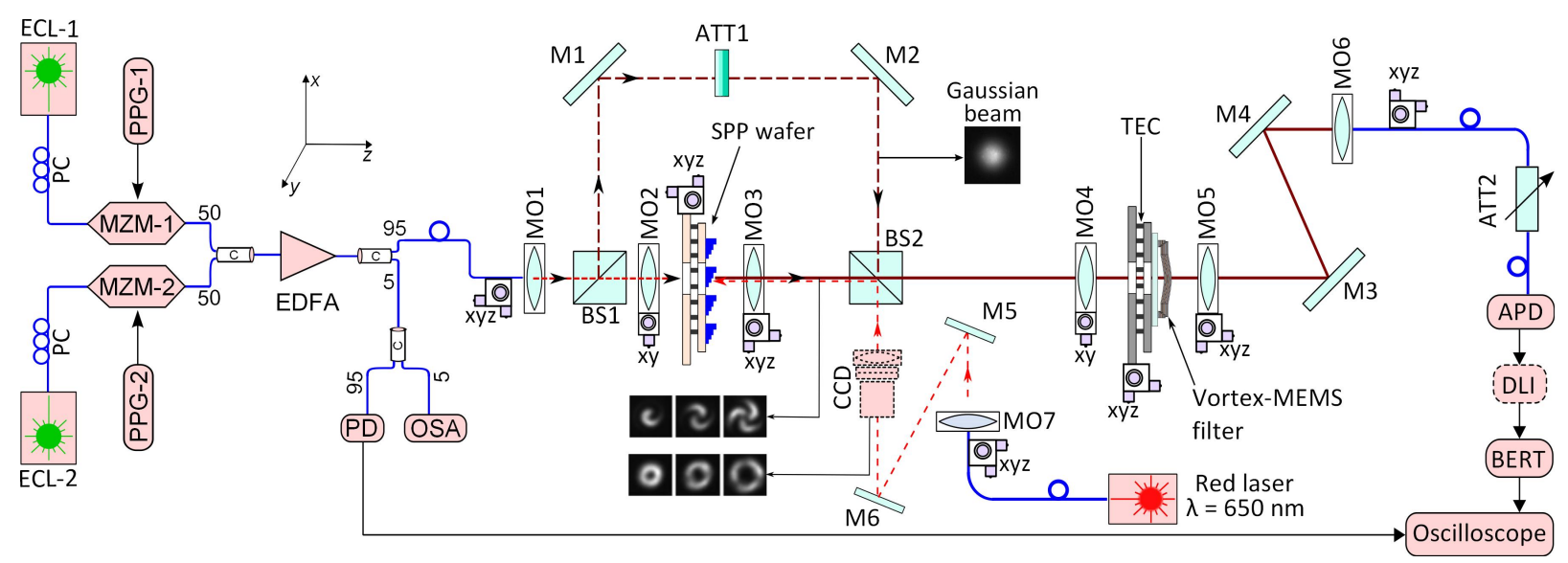

Figure 10: Setup used the BER measurements.

dBm. Purposely no optical band-pass filter is used after EDFA for filtering of amplified spontaneous emission (ASE), as it is to be filtered by the MEMS-filter. Pre-amplified optical signal, collimated from the fiber output by microscope objective MO1, is split by beam splitter BS1 to generate two copies of the WDM signal. Then one signal copy, being passed through SPP, acquires the OAM corresponding to the azimuthal order of SPP, whereas another one remains Gaussian. SPPs at the transmitter have the same construction as SPPs deposited on the apertures of MEMS filters, but are deposited directly on the Si substrate. The input beam is incident to SPP from the side of substrate, where an ARC is applied. MO2 serves to match the beam waist with the SPP, and MO3 collimates the output vortex beam. A path difference of $\sim 35 \mathrm{~cm}$ between Gaussian beam and vortex beam provides 1.17 ns delay between OAM-multiplexed channels. BS2 provides multiplexing of Gaussian and OAM beams, forming four independent information channels. Variable attenuator ATT1 allows for adjusting the ratio between powers transmitted in Gaussian and OAM channels. First, it is required for compensation of higher losses in the path of vortex channel compared with that of Gaussian channel due to SPP and MO2, MO3. Moreover, as the experiments show, power in the Gaussian channel should be additionally reduced to some extent relative to the vortex channel in order to provide stable detection of both these spatial channels. This can be explained by different efficiency of beam coupling to the MEMS filters for Gaussian and OAM mode. The position of ATT1 is kept the same when receiving the Gaussian and OAM channels and their power difference after BS2 is kept at $1.45 \mathrm{~dB}$ throughout all the experiments.

For preliminary alignment of the SPP with the input IR beam, the fiber coupled red light laser is used, which points the SPP of proper azimuthal order on the wafer. The beam from the red light laser, previously aligned with the IR beam by means of mirrors M5 and M6, is also transmitted through the beam splitter BS2. For precise alignment of the SPPs, intensity distribution of its output beam is monitored using a phosphor-coated near IR CCD-camera, placed after BS2. Also the use of CCD-camera facilitates alignment of OAM-MUX in order to have Gaussian and vortex channels propagating parallel over the same spatial free space link. As the elements BS1, BS2, M1 and M2 form a Mach-Zehnder interferometer, it is convenient to control coincidence of Gaussian and vortex channels from the fringe patterns at the output of OAM-MUX see in Fig. 10). When aligning OAM-MUX, its feeding fiber is connected directly to the laser without modulators and WDM-MUX to have coherent radiation for contrast fringe patterns. The multiplexed signal is transmitted over a short $(23 \mathrm{~cm})$ free space link. At the receiver side the multiplexed signal is focused by MO4, which matches the beam waist with the aperture of MEMS-filter, and incident to the SPP. The azimuthal order of SPP corresponds to the opposite topological charge of the vortex beam when receiving the OAM channel, and the MEMS-filter without SPP is used when receiving the Gaussian channel. Tuning of the filters is realized by controlling the substrate temperature with a thermoelectric cooler (TEC) element. MO5 provides collimation of the beam transmitted through the MEMS-filter, and MO6 provides coupling of this beam to the standard single mode fiber (SSMF), which serves as a spatial filter supporting only the Gaussian mode, and also as a link to the photo-detector. Mirrors M3, M4 provide alignment of the MEMS-filter output beam to the fiber. Detection of the received signal 

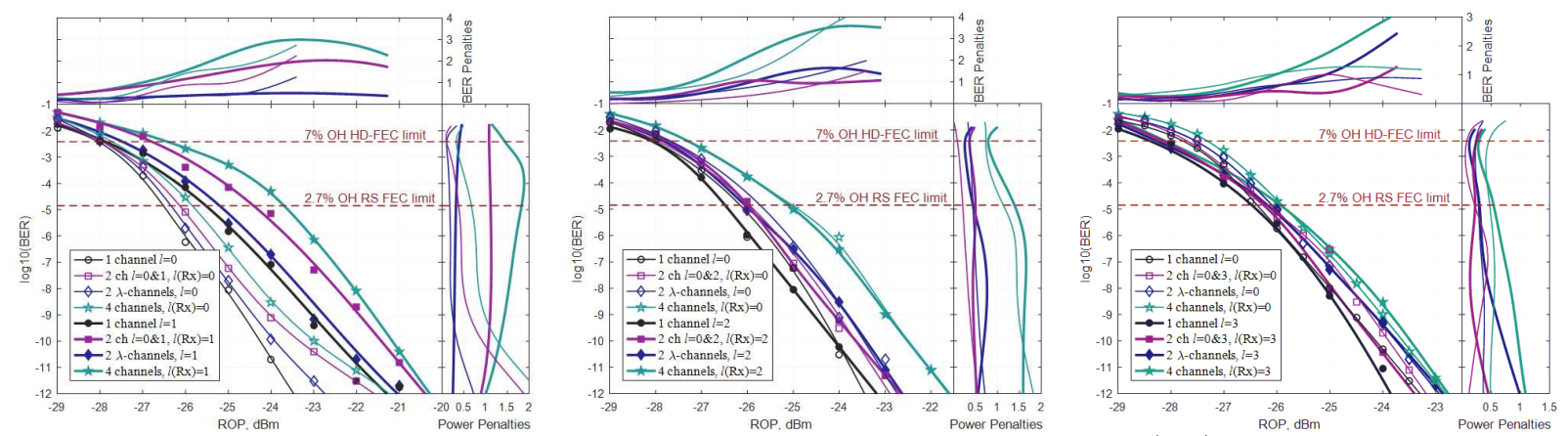

Figure 11: BER vs. ROP curves, and BER and power penalties for OOK (a-c) modulation format when demultiplexing wavelength and OAM channels. The graphs correspond to transmission of the Gaussian channel and the OAM channel of orders: $l_{p}=1$ (left), $l_{p}=2$ (center) and $l_{p}=3$ (right), respectively.
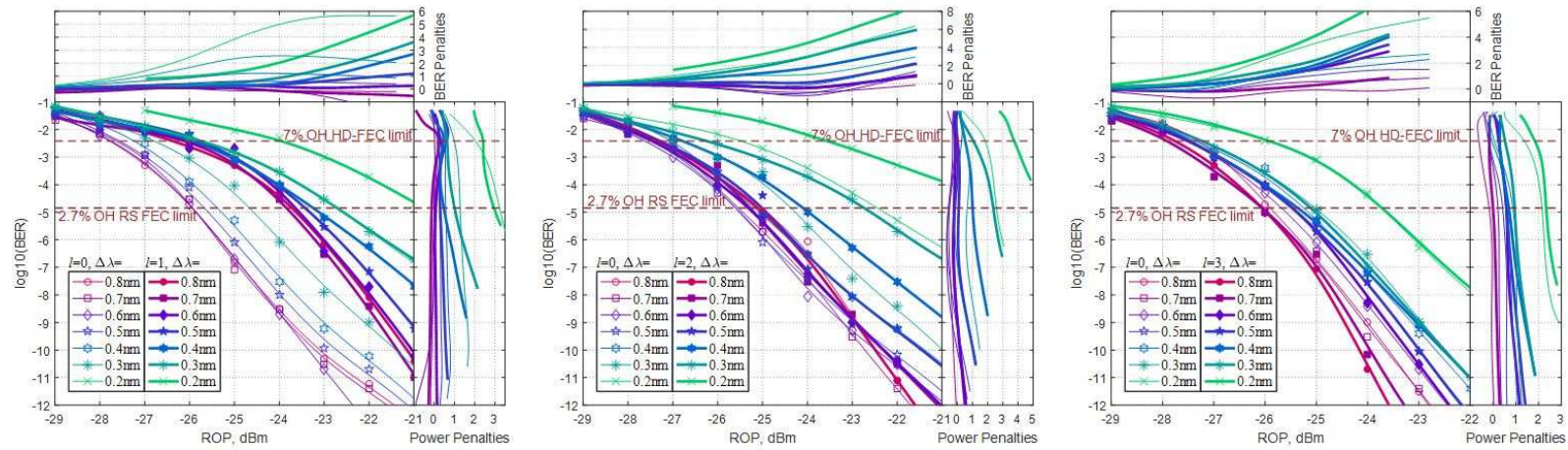

Figure 12: BER vs. ROP curves, and BER and power penalties for OOK modulation when four (two wavelength and two OAM) channels are being transmitted for different spacing between wavelength channels and the received channel has higher wavelength. Each graph corresponds to transmission of the Gaussian channel and the OAM channel of order $l=1$ (left), $l=2$ (center) and $l=3$ (right).

is provided by an avalanche photodiode (APD).

\section{DIGITAL DATA TRANSMISSION}

The experimental set-up allows us to measure BER for different number of information channels in order to determine the penalties when simultaneous WDM and OAM multiplexing/demultiplexing is performed. Thus, four different cases are considered: one channel (reference BER curve for each wavelength and each OAM alone), two spatial channels at the same wavelength but different OAMs, two wavelength channels without extra OAM multiplexing, and four channels where two spatial and two wavelength channels are combined (Fig. 12). The series of BER measurements mentioned above is implemented in both the OAM channel and Gaussian channel, using for demultiplexing the filters with and without SPP respectively. During the measurements the received channel has the wavelength in the range from 1536 to $1544 \mathrm{~nm}$. The spacing between channels in wavelength domain is fixed and equals $\Delta \lambda=0.8 \mathrm{~nm}$.

In case of two transmitted OAM channels, the measured one is tagged as $(\mathrm{Rx})$. For example, when two channels with $l=0$ and $l=1$ are being transmitted at the same wavelength, the sign $l(\mathrm{Rx})=0$ means that the Gaussian channel is measured, and the sign $l(\mathrm{Rx})=1$ denotes measuring of the OAM channel. This notation is assumed for all figures. In order to verify that OAM/wavelength multiplexing with proposed devices is transparent to modulation formats, the same sets of BER curves vs. Received Optical Power (ROP) have been collected for OOK (Fig. 12 a-c). We do not use amplifiers at the receiver side in order to avoid the noise due to ASE. As defined in optical transmission network (OTN) standard, BER below $3.8 \times 10^{-3}$ is required for successful transmission using Forward Error Correction (FEC) with 7\% overhead (OH). However, this BER threshold 

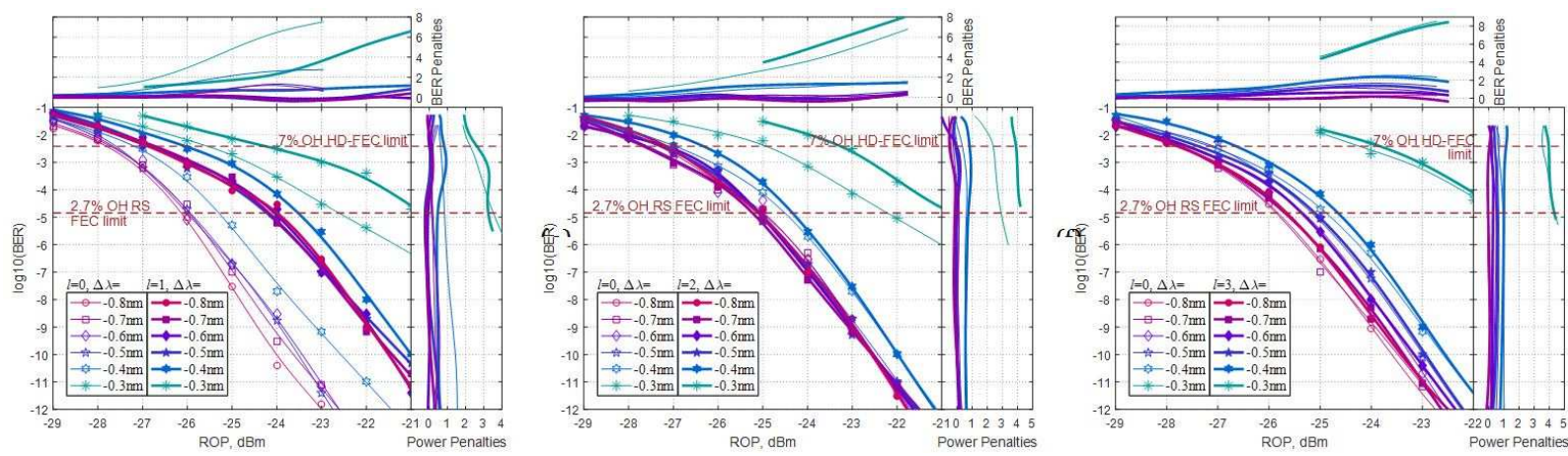

Figure 13: BER vs. ROP curves, and BER and power penalties for OOK modulation when four (two wavelength and two OAM) channels are being transmitted for different spacing between wavelength channels and the received channel has lower wavelength. Each graph corresponds to transmission of the Gaussian channel and the OAM channel of order $l=1$ (left), $l=2$ (center) and $l=3$ (right).

Table 2: Maximum BER and ROP penalties at FEC BER thresholds for multiplexing four channels, $\Delta \lambda=0.4 \mathrm{~nm}$ relative to $\Delta \lambda=0.8, \mathrm{~nm}$.

\begin{tabular}{c|l|l|l|l|l}
\multicolumn{2}{c|}{ BER threshold } & \multicolumn{2}{c|}{$7 \%$ OH HD-FEC } & \multicolumn{2}{c}{$2.7 \%$ OH RS FEC } \\
\hline \multicolumn{2}{c}{ Penalties } & $\log 10(\mathrm{BER})$ & ROP $(\mathrm{dB})$ & $\log 10(\mathrm{BER})$ & ROP $(\mathrm{dB})$ \\
\hline$l(\mathrm{Tx})=0$ and 1 & $l(\mathrm{Rx})=0$ & 0.66 & 0.62 & 1.96 & 0.84 \\
\hline & $l(\mathrm{Rx})=1$ & 0.60 & 0.83 & 0.80 & 0.48 \\
\hline$l(\mathrm{Tx})=0$ and 2 & $l(\mathrm{Rx})=0$ & 0.31 & 0.35 & 0.96 & 0.63 \\
\hline & $l(\mathrm{Rx})=2$ & 0.86 & 0.84 & 1.63 & 1.00 \\
\hline$l(\mathrm{Tx})=0$ and 3 & $l(\mathrm{Rx})=0$ & 0.52 & 0.62 & 1.98 & 0.94 \\
\hline & $l(\mathrm{Rx})=3$ & 1.00 & 1.15 & 2.12 & 1.00
\end{tabular}

seems not reasonable for the scenarios of short-reach optical links and data center interconnects because of large overhead and high latency of FEC. The recently accepted IEEE 802.3bm standard implies FEC with $2.7 \%$ OH Reed-Solomon (RS) code with $100 \mathrm{~ns}$ latency. This type of FEC requires BER threshold at $1.42 \times 10^{-5}$ to achieve output BER lower than 10-15.17,18 Therefore, at the BER graphs for OOK modulation the BER limit at $1.42 \times 10^{-5}$ is also considered. To assess the performance of transmission system when demultiplexing wavelength and OAM channels with our vortex-MEMS filters, we calculate the BER and ROP penalties over the whole tested range of ROP. From Fig. 12 the general conclusion can be made that the closer azimuthal orders of the transmitted OAM channels, the higher BER and power penalties. For less OAM separation the BER and power penalties increase as can be seen in Fig. 12 from (a) to (c). In order to assess the selectivity of the vortexMEMS filters in the wavelength domain, we measured performance of the system for different spacing between wavelength channels in case of OOK modulation (Figs. 13,14). The devices were tested for the fully loaded system, i.e. simultaneous multiplexing of two wavelength and two OAM channels, and $\Delta \lambda$ was decreasing form $0.8 \mathrm{~nm}$ with the step of $0.1 \mathrm{~nm}$ while the wavelength channels remained separable. As before, values of BER in both OAM channel and Gaussian channel were measured. BER and power penalties were calculated relative to the case of largest spacing $\Delta \lambda=0.8 \mathrm{~nm}$. As the MEMS filter proves to possess non-symmetric bandwidth at the resonance wavelength (with increasing the wavelength the transmittance increases smoothly up to the resonance and then falls down abruptly), two scenarios were tested: when the influencing channel has the lower (Fig. 13) and the higher (Fig. 14) wavelength relative to the received channel. It is clearly seen that the MEMS-filter suppresses the non-resonant channel differently depending on the positioning of the suppressed channel. The performance degradation due to crosstalk between wavelength channels becomes distinguishable for $\Delta \lambda \leq 0.5 \mathrm{~nm}$ in the case when the received channel has higher wavelength than the filtered channel, and for $\Delta \lambda \leq 0.4 \mathrm{~nm}$ when the received channel has lower wavelength than the filtered channel. But in the former case the received signal remains detectable with the BER values lower than $7 \%$ OH FEC threshold even with $\Delta \lambda=0.2 \mathrm{~nm}$, while in the latter case the quality of the received signal degrades rapidly with diminishing the spacing between wavelength 
channels beyond $0.4 \mathrm{~nm}$. Nevertheless, wavelength channels in the latter case still can be demultiplexed when $\Delta \lambda$ $=0.3 \mathrm{~nm}$. Therefore, the overall behavior of MEMS-filters in wavelength domain suggests its reasonable usage with the $50 \mathrm{GHz}$ DWDM frequency grid. ${ }^{19}$ Table 2 summarizes maximum BER and ROP penalties (among both placement scenarios of wavelength channels mentioned above) when multiplexing the Gaussian beam and OAM beams of azimuthal orders $l=1,2$ and 3. Thus, from Figs. 12-14 the summarizing conclusion can be drawn, that the behavior of the vortex-MEMS filters in both wavelength and OAM domains is similar the larger spacing between channels in each multiplexing domain the better the transmission system performance (lower BER and power penalties), and vice versa.

\section{CONCLUSION}

In this paper, we demonstrated a new wavelength-tunable micro-component for simultaneous selection of the waves with different wavelengths and values of OAM MEMS-based Fabry-Perot filter with integrated spiral phase plate. The proposed device is suitable for dense on-chip integration and is dedicated for the next generation optical links in both long-haul and short-range scenarios, simultaneously utilizing all three degrees of freedom of the electromagnetic waves: wavelength, polarization, and OAM. Experimental measurements of system performance when multiplexing two wavelength channels (with $0.8 \mathrm{~nm}$ spacing) and two OAM channels (Gaussian beam is combined with OAM beams of azimuthal orders $l=1,2,3$ ) demonstrate, that in the case of intensity modulation power penalties do not exceed $1.45 \mathrm{~dB}$ and $1.83 \mathrm{~dB}$ at the $7 \%$ OH HD-FEC and $2.7 \%$ $\mathrm{OH}$ Reed-Solomon FEC BER thresholds respectively. With the ROP up to $-20 \mathrm{dBm}$ the error-free transmission $\left(\mathrm{BER}<10^{-12}\right)$ is demonstrated. Moderate BER/ROP penalties when shifting wavelength channels as close as 0.4 $\mathrm{nm}$ (1 order/1.15 dB and 2.12 orders $/ 1 \mathrm{~dB}$ at the above mentioned BER thresholds respectively) suggest usage of the proposed devices with the $50 \mathrm{GHz}$ DWDM frequency grid. At the same time, transmission tests when

multiplexing Gaussian beam with OAM beams of different orders demonstrate the behavior of the vortex-MEMS filters in OAM domain generally similar to that in the wavelength domain - the larger the distance between multiplexed channels, the better the transmission system performance.

\section{ACKNOWLEDGMENTS}

V. S. L. acknowledges the support of DAAD and the Ministry of Education and Science of Russian Federation within the joint program "Michail Lomonosov". M. F. S. and M. W. acknowledge support from the Helmholtz program "Science and Technology of Nanosystems (STN)" and from the "Karlsruhe School of Optics and Photonics (KSOP)". The authors acknowledge Y. Hopf and F. Lenze for their contribution to setup.

\section{REFERENCES}

1. A. E. Willner, H. Huang, Y. Yan, Y. Ren, N. Ahmed, G. Xie, C. Bao, L. Li, Y. Cao, Z. Zhao, J. Wang, M. P. J. Lavery, M. Tur, S. Ramachandran, A. F. Molisch, N. Ashrafi, and S. Ashrafi, "Optical communications using orbital angular momentum beams," Adv. Opt. Photon. 7, 66-106 (2015).

2. L. Allen, M. Beijersbergen, R. Spreeuw, and J. Woerdman, "Orbital angular momentum of light and the transformation of Laguerre-Gaussian laser modes," Phys. Rev. A 45, 8185-8189 (1992).

3. J. Wang, J.-Y. Yang, I. M. Fazal, N. Ahmed, Y. Yan, H. Huang, Y. Ren, Y. Yue, S. Dolinar, M. Tur, and A. E. Willner, "Terabit free-space data transmission employing orbital angular momentum multiplexing," Nat. Photon. 6(7), 488-496 (2012).

4. G. Gibson, J. Courtial, M. Padgett, M. Vasnetsov, V. Pasko, S. Barnett, and S. Franke-Arnold, "Free-space information transfer using light beams carrying orbital angular momentum," Opt. Express 12, 5448-5456 (2004).

5. H. Li, D. Phillips, X. Wang, Y. Ho, L. Chen, X. Zhou, J. Zhu, S. Yu, and X. Cai, "Orbital angular momentum vertical-cavity surface-emitting lasers," Optica 2(6), 547-552 (2015).

6. W. Zhang, H. Wang, and K. Bergman, "Next-generation optically interconnected high-performance data centers," J. Lightw. Technol. 30(24), 3836-3844 (2012).

7. M. A. Taubenblatt, "Optical interconnects for high-performance computing," J. Lightw. Technol. 30(4), 448457 (2012). 
8. S. Paul, V. S. Lyubopytov, M. F. Schumann, J. Cesar, A. Chipouline, M. Wegener, and F. Kueppers, "Wavelength-selective orbital-angular-momentum beam generation using MEMS tunable Fabry-Perot filter," Opt. Lett. 41(14), 32493252 (2016).

9. S. Paul, C. Gierl, J. Cesar, M. Malekizandi, C. Neumeyr, M. Ortsiefer, and F. Küppers, "10-Gb/s Direct Modulation of Widely Tunable 1550-nm MEMS VCSEL," IEEE J. Sel. Top. Quantum Electron. 21(6), $436-443$ (2015).

10. M. Schumann, T. Buckmann, N. Gruhler, M. Wegener, and W. Pernice, Wolfram, "Hybrid 2D-3D optical devices for integrated optics by direct laser writing," Light Sci. Appl. 3, e175 (2014).

11. H. Huang, Y. Ren, N. Ahmed, Y. Yan, Y. Yue, A. Bozovich, J.-Y. Yang, K. Birnbaum, J. Choi, B. Erkmen, S. Dolinar, M. Tur, and A. Willner, "Demonstration of OAM Mode Distortions Monitoring using InterferenceBased Phase Reconstruction," in CLEO, OSA Technical Digest (online) (OSA, 2012), paper CF3C.4.

12. J. Wang, M. J. Padgett, S. Ramachandran, M. P. J. Lavery, H. Huang, Y. Yue, N. Bozinovic, S. E. Golowich, and A. E. Willner, in Optical Fiber Telecommunications VIB, (Academic, 2013), Chap. 12.

13. Y. Yan, G. Xie, M. P. J. Lavery, H. Huang, N. Ahmed, C. Bao, Y. Ren, Y. Cao, L. Li, Z. Zhao, A. F. Molisch, M. Tur, M. J. Padgett, and A. E. Willner, "High-capacity millimetre-wave communications with orbital angular momentum multiplexing," Nat. Commun. 5, 4876 (2014).

14. J. Wang, S. Li, C. Li, L. Zhu, C. Gui, D. Xie, Y. Qiu, Q. Yang, and S. Yu, "Ultra-high 230-bit/s/Hz spectral efficiency using OFDM/OQAM 64-QAM signals over polmuxed 22 orbital angular momentum (OAM) modes," in Proceedings of Optical Fiber Communication Conference (OFC), paper W1H.4 (2014).

15. H. Huang, G. Xie, Y. Yan, N. Ahmed, Y. Ren, Y. Yue, D. Rogawski, M. J. Willner, B. I. Erkmen, K. M. Birnbaum, S. J. Dolinar, M. P. J. Lavery, M. J. Padgett, M. Tur, and A. E. Willner, "100 Tbit/s freespace data link enabled by three-dimensional multiplexing of orbital angular momentum, polarization, and wavelength," Opt. Lett. 39(2), 197-200 (2014).

16. J. Wang, S. Li, M. Luo, J. Liu, L. Zhu, C. Li, D. Xie, Q. Yang, S. Yu, J. Sun, X. Zhang, W. Shieh, and A. E. Willner, "N-dimentional multiplexing link with 1.036-Pbit/s transmission capacity and 112.6-bit/s/Hz spectral efficiency using OFDM-8QAM signals over 368 WDM pol-muxed 26 OAM modes," in Proceedings of European Conference and Exhibition on Optical Communication (ECOC), paper Mo.4.5.1 (2014).

17. "IEEE standard for ethernet - amendment 3: physical layer specifications and management parameters for $40 \mathrm{~Gb} / \mathrm{s}$ and $100 \mathrm{~Gb} / \mathrm{s}$ operation over fiber optic cables, IEEE Std 802.3bm-2015 (Amendment to IEEE Std 802.3-2012 as amended by IEEE Std 802.3bk-2013 and IEEE Std 802.3bj-2014), pp. 1172, March 2015.

18. J. Petrilla, "100G SR4 \& RS $(528,514,7,10)$ FEC," Avago Technologies, Tech. Rep., September 2012.

19. Recommendation ITU-T G.694.1 (02/2012) "Spectral grids for WDM applications: DWDM frequency grid." 